Article

\title{
Barriers to the Use of Trastuzumab for HER2+ Breast Cancer and the Potential Impact of Biosimilars: A Physician Survey in the United States and Emerging Markets
}

\author{
Philip Lammers ${ }^{1, *}$, Carmen Criscitiello ${ }^{2}$, Giuseppe Curigliano ${ }^{2}$ and Ira Jacobs $^{3}$ \\ 1 Meharry Medical College, 1005 Dr. D.B. Todd, Jr. Boulevard, Nashville, TN 37208-3599, USA \\ 2 Early Drug Development for Innovative Therapies Division, European Institute of Oncology, \\ Via Ripamonti, 435-20141 Milano, Italy; E-Mails: carmen.criscitiello@ieo.it (C.C.); \\ giuseppe.curigliano@ieo.it (G.C.) \\ 3 Pfizer Inc, 235 East 42nd St, New York, NY 10017-5755, USA; E-Mail: ira.jacobs@pfizer.com \\ * Author to whom correspondence should be addressed; E-Mail: plammers@mmc.edu; \\ Tel: +1-615-327-5948; Fax: +1-615-327-6733.
}

Received: 3 June 2014; in revised form: 10 September 2014 / Accepted: 12 September 2014 /

Published: 17 September 2014

\begin{abstract}
Trastuzumab in combination with chemotherapy has become a standard of care for patients with HER2+ breast cancer. The cost of therapy, however, can limit patient access to trastuzumab in areas with limited financial resources for treatment reimbursement. This study examined access to trastuzumab and identified potential barriers to its use in the United States, Mexico, Turkey, Russia and Brazil via physician survey. The study also investigated if the availability of a biosimilar to trastuzumab would improve access to and use of HER2 monoclonal antibody therapy. Across all countries, a subset of oncologists reported barriers to the use of trastuzumab in a neoadjuvant, adjuvant or metastatic setting. Common barriers to the use of trastuzumab included issues related to insurance coverage, drug availability and cost to the patient. Overall, nearly half of oncologists reported that they would increase the use of HER2 monoclonal antibody therapy across all treatment settings if a lower cost biosimilar to trastuzumab were available. We conclude that the introduction of a biosimilar to trastuzumab may alleviate cost-related barriers to treatment and could increase patient access to HER2-directed therapy in all countries examined.
\end{abstract}

Keywords: trastuzumab; biosimilar; access; emerging markets; HER2+ breast cancer 


\section{Introduction}

Breast cancer is the most common cancer among women and is the primary cause of cancer death among women worldwide, accounting for $\sim 522,000$ deaths in 2012 [1]. Incidence rates differ throughout the world, but rates are highest in developed countries [1]. In the United States (US), for example, over 235,000 new cases of invasive breast cancer are expected in 2014 [2]. Survival rates also vary across regions; rates are over $80 \%$ in North America, Australia, Sweden, Finland and Japan, $\sim 60 \%$ in Brazil, Poland and Slovakia, but below 40\% in Algeria [3]. Low survival rates in less-developed countries may be explained by a lack of early detection programs and poor access to the newest therapies [4]. Even in the U.S., despite advanced detection and treatment programs, it is estimated that approximately 40,000 women will die from breast cancer in 2014 [2].

Trastuzumab, marketed under the trade name, Herceptin ${ }^{\circledR}$ (Genentech, South San Francisco, CA, USA), is a monoclonal antibody directed against the human epidermal growth factor receptor 2 protein (HER2) [5]. Trastuzumab is indicated as an adjuvant treatment for HER2-overexpressing breast cancer and for the treatment of metastatic HER2-overexpressing breast cancer [5,6]. In three large-scale, randomized, open-label trials in women with HER2+ breast cancer, treatment with adjuvant trastuzumab resulted in a $52 \%$ increase in disease-free survival $[7,8]$ and a $33 \%$ reduction in risk of death [8] relative to chemotherapy alone. Likewise, the addition of trastuzumab to standard chemotherapy in patients with first-line metastatic HER2+ breast cancer resulted in an increase in time to disease progression and resulted in a $20 \%$ decrease in the risk of death [9]. Trastuzumab, in combination with pertuzumab and docetaxel, has also shown efficacy in women with locally-advanced, inflammatory or early HER2+ breast cancer [10].

Biologics, such as trastuzumab, are complex medicines developed from living organisms [11]. The cost associated with biologic therapy may limit patient access to these medicines in areas with limited financial resources for treatment reimbursement. Decreasing the cost of therapy and, as a result, improving access to biologic treatments is a priority in such areas. The use of biosimilars may be one approach to increase access to specific biologic therapies by offering a comparable, but more affordable, alternative. The term "biosimilar" refers to a biologic drug that is developed to be highly similar, in terms of safety, purity and potency, to an existing licensed reference biologic [11-13]. Biosimilars treat the same conditions as the reference biologic, but are commonly priced $25 \%$ to $30 \%$ less $[12,14]$. The use of biosimilars is expected to grow substantially in the near future as biologic patents expire [12,13]. In anticipation of this growth and in recognition of the potential that biosimilars have to increase access to biologic therapy, the Biologics Price Competition and Innovation Act of 2009 (part of the Patient Protection and Affordable Care Act) and the European Medicines Agency Committee for Medicinal Products for Human Use have established guidelines for the development and streamlined approval of biosimilars in the U.S. [15] and the EU [16], respectively.

Access to trastuzumab may be a barrier to effective treatment for a large group of patients. With this issue in mind, the objectives of the current study were to examine the level of access that physicians and patients in the U.S., Mexico (MEX), Turkey (TUR), Russia (RUS) and Brazil (BRZ) have to Herceptin (trastuzumab) and to identify potential barriers that may prevent its use in the treatment of HER2+ breast cancer. An additional objective of this study was to determine whether the introduction of a 
biosimilar to trastuzumab would increase access to and use of HER2 monoclonal antibody therapy in these countries.

\section{Experimental Section}

\subsection{Approach}

A questionnaire was designed to evaluate the access to and usage of trastuzumab by oncologists in the U.S., BRZ, MEX, RUS and TUR. Recruiters who were familiar with each country's healthcare system and culture engaged physicians. Physicians were identified from proprietary databases, association lists, telephone directories and other commercially available sources. Alternatively, physicians who were existing members of a recruiter's online community could request to participate in the survey. Physicians were invited to participate by telephone or email. Those agreeing to participate were emailed a link to the automated online survey. A short screener survey was used to identify physicians for the main survey. To participate, physicians must have been a full-time hematologist/medical oncologist (practicing at least $30 \mathrm{~h} /$ week), in practice between 2 and 30 years, spent at least 75\% of the time in direct patient care and have a practice consisting of a combination of hospital and office based (U.S. only), and at least $20 \%$ of their cancer patients must have been diagnosed with breast cancer (at least $10 \%$ of those patients being HER2+).

The goal was to survey 500 physicians; 200 in the U.S. and 75 each in BRZ, MEX, RUS and TUR. In total, 7,994 physicians were invited to participate in the survey (U.S. $=1,530$, BRZ $=3,848$, MEX $=1,529$, RUS $=702$, TUR $=385$ ). The short screener survey was failed by 351 physicians, and another 16 physicians started the main survey, but discontinued without completing. Once a country achieved its pre-specified goal, the survey was no longer administered in that country, regardless of the number of invited physicians remaining/willing to participate. Using these sample sizes, the margins of error at the $95 \%$ confidence level were $\pm 4.4 \%$ (overall), $\pm 6.9 \%$ (U.S.; $n=200$ ) and $\pm 11.3 \%$ (all other countries; $n=75)$.

\subsection{Survey Details}

The questionnaire focused on HER2+ breast cancer patients. Specific questions were related to: breast cancer disease categories; types of patient insurance; factors considered when making treatment decisions; use of treatment guidelines; use of trastuzumab; barriers to the use of trastuzumab; and the potential use of a less expensive biosimilar version of trastuzumab if it were available. Questions were answered in a variety of forms, including: yes/no; percentage; 5- or 7-point Likert scale; and select all that apply. In some cases, participants were offered a text box to further explain their answers. The survey was expected to take $\sim 10$ min to complete, and participants received an honorarium upon completion. The survey was administered from December, 2012, to January, 2013. 


\section{Results}

\subsection{Breast Cancer Patient Population}

Physicians reported that $41 \%$ (range, 36\%-53\%) of their patients had breast cancer, and nearly one-third (range, 25\%-35\%) of the cancers were characterized as HER2+ (Table 1). HER2+ breast cancer patients utilized different types of insurance coverage across markets. More than $50 \%$ of patients, particularly those in MEX, TUR and RUS, had government-funded insurance (overall $=55 \%$, U.S. $=37 \%, \mathrm{BRZ}=34 \%, \mathrm{MEX}=69 \%$, TUR $=93 \%$, RUS $=74 \%)$. Twenty-two percent of patients received private medical insurance through their employer (U.S. $=38 \%$, BRZ $=26 \%, \mathrm{MEX}=8 \%$, TUR $=4 \%$, RUS $=7 \%$, while $18 \%$ (U.S. $=20 \%, \mathrm{BRZ}=36 \%, \mathrm{MEX}=16 \%$, TUR $=2 \%, \mathrm{RUS}=8 \%$ ) paid for their own private insurance. Patients in MEX and RUS were the most likely to pay for medical expenses out of pocket, compared with other markets (overall $=5 \%$, U.S. $=5 \%, \mathrm{BRZ}=5 \%$, MEX $=8 \%$, TUR $<1 \%$, RUS $=11 \%$ ).

Table 1. Description of the breast cancer patient population.

\begin{tabular}{ccccccc}
\hline Type of Cancer & Overall & U.S. & BRZ & MEX & TUR & RUS \\
\hline Breast, \% of all cancer patients & 41 & 36 & 41 & 53 & 36 & 51 \\
HER2+, \% of breast cancer patients & 31 & 35 & 27 & 28 & 25 & 33 \\
\hline
\end{tabular}

U.S., United States; BRZ, Brazil; MEX, Mexico; TUR, Turkey; RUS, Russia.

\subsection{Factors Considered When Treating HER2+ Breast Cancer Patients}

Physicians reported that clinical treatment guidelines (88\%), goals of therapy (68\%) and recommendations from opinion leaders $(52 \%)$ were the factors most considered when making treatment decisions regarding patients with HER2+ breast cancer. These were followed by performance status (49\%), patient insurance status (39\%), patient demographics $(25 \%)$ and geographic locale $(8 \%)$. National Comprehensive Cancer Network ( $\mathrm{NCCN})$ guidelines were regarded as the most influential treatment guidelines among all physicians (overall $=66 \%$ ). NCCN guidelines are considered by $82 \%$ of all physicians (U.S. $=92 \%, \mathrm{BRZ}=95 \%, \mathrm{MEX}=84 \%$, TUR $=97 \%$, RUS $=25 \%$ ) when treating patients. American Society of Clinical Oncology (overall $=58 \%$, U.S. $=47 \%, \mathrm{BRZ}=89 \%, \mathrm{MEX}=76 \%$, TUR $=61 \%$, RUS $=36 \%$ ), European Society for Medical Oncology (overall $=56 \%$, U.S. $=$ NA, $\mathrm{BRZ}=63 \%, \mathrm{MEX}=36 \%, \mathrm{TUR}=65 \%$, RUS $=59 \%$ ) and St. Gallen (overall $=47 \%$, U.S. $=$ NA, $\mathrm{BRZ}=63 \%, \mathrm{MEX}=59 \%, \mathrm{TUR}=32 \%$, RUS $=33 \%$ ) guidelines were also frequently reported as guidelines considered by physicians. National Ministry of Health guidelines were reported by $52 \%$ of physicians in RUS.

Physicians were asked whether the clinical guidelines they follow explicitly recommend the use of trastuzumab for the treatment of HER2+ breast cancer patients in different clinical settings. Across all countries, a majority of physicians reported that trastuzumab is explicitly recommended for use in a neoadjuvant setting in HER2+ breast cancer patients (overall $=57 \%$ ). This was reported by a greater number of physicians in the U.S. (66\%), TUR (72\%) and RUS (56\%) and fewer in BRZ (35\%) and MEX (39\%). Across all countries, a majority of physicians reported that trastuzumab is also explicitly recommended for use in an adjuvant setting in HER2+ breast cancer patients if the therapy duration is 
greater than 6 months (overall $=63 \%$ ). This was reported by a greater number of physicians in the U.S. (69\%), TUR (81\%) and RUS (65\%) than in BRZ (49\%) and MEX (44\%). Finally, a majority of physicians reported that trastuzumab is explicitly recommended for use in a metastatic setting in HER2+ breast cancer patients. This was consistent for both first line (overall $=68 \%$, U.S. $=77 \%, \mathrm{BRZ}=55 \%$, $\mathrm{MEX}=49 \%, \mathrm{TUR}=87 \%, \mathrm{RUS}=56 \%)$ and second line (overall $=64 \%$, U.S. $=71 \%, \mathrm{BRZ}=48 \%$, MEX $=41 \%$, TUR $=83 \%$, RUS $=68 \%$ ) treatment settings.

\subsection{Trastuzumab Usage and Barriers to Treatment}

Physicians were asked to report how frequently $(1=$ always, $2=$ frequently, $3=$ not so often, $4=$ rarely, 5 = never) they used trastuzumab in HER2 + breast cancer patients in different clinical settings. In all countries, except RUS, a majority of physicians reported always or frequently using trastuzumab in a neoadjuvant setting (overall $=73 \%$, U.S. $=78 \%$, BRZ $=80 \%$, MEX $=81 \%$, TUR $=83 \%$, RUS $=33 \%$ ). A majority of physicians also reported always or frequently using trastuzumab in an adjuvant (overall $=92 \%$, U.S. $=93 \%, \mathrm{BRZ}=99 \%, \mathrm{MEX}=95 \%$, TUR $=97 \%$, RUS $=74 \%)$ and in a metastatic (overall $=92 \%$, U.S. $=94 \%$, BRZ $=100 \%$, MEX $=93 \%$, TUR $=98 \%$, RUS $=73 \%$ ) setting. Physicians in RUS reported more infrequent use of trastuzumab in these settings than physicians from any other country.

Physicians who reported a score of 3 (not so often), 4 (rarely) or 5 (never) were asked to identify the reasons they did not use trastuzumab in different clinical settings (Table 2). Across all countries, the most common barriers to the use of trastuzumab in a neoadjuvant setting were related to insurance coverage, availability of the drug, treatment guidelines, patient comorbidities and cost to the patient. The most common barriers to the use of trastuzumab in an adjuvant setting were related to availability of the drug, cost to the patient, patient comorbidities, clinical data, insurance coverage and treatment guidelines. The most common barriers to the use of trastuzumab in a metastatic setting were related to insurance coverage, availability of the drug and treatment guidelines.

Table 2. Most common barriers to the use of trastuzumab ( $n(\%)$ of physicians) $)^{\mathrm{a}, \mathrm{b}}$.

\begin{tabular}{lcccccc}
\hline In a Neoadjuvant Setting & $\begin{array}{c}\text { Overall } \\
(\boldsymbol{n}=\mathbf{1 3 7})\end{array}$ & $\begin{array}{c}\text { U.S. } \\
(\boldsymbol{n}=\mathbf{4 5})\end{array}$ & $\begin{array}{c}\text { BRZ } \\
(\boldsymbol{n}=\mathbf{1 5})\end{array}$ & $\begin{array}{c}\text { MEX } \\
(\boldsymbol{n}=\mathbf{1 4})\end{array}$ & $\begin{array}{c}\text { TUR } \\
(\boldsymbol{n}=\mathbf{1 3})\end{array}$ & $\begin{array}{c}\text { RUS } \\
(\boldsymbol{n}=\mathbf{5 0})\end{array}$ \\
\hline $\begin{array}{l}\text { Not included in the formulary of drugs covered by } \\
\text { patients' insurance }\end{array}$ & 38 & 33 & 53 & 50 & 23 & 62 \\
$\begin{array}{l}\text { Not available in the hospital/clinic where I practice } \\
\text { Use not recommended by treatment guidelines or }\end{array}$ & 37 & 11 & 33 & 50 & 23 & 42 \\
$\begin{array}{l}\text { protocol I follow in this setting } \\
\begin{array}{l}\text { Patient has medical issues why they cannot take the } \\
\text { drug (e.g., cardiac) }\end{array}\end{array}$ & 37 & 49 & 40 & 36 & 0 & 38 \\
High out-of-pocket treatment cost for patient & 34 & 31 & 47 & 21 & 23 & 16 \\
\hline $\begin{array}{l}\text { In an Adjuvant Setting } \\
\text { Overall }\end{array}$ & $\begin{array}{c}\text { U.S. } \\
(\boldsymbol{n}=\mathbf{4 1})\end{array}$ & $\begin{array}{c}\text { BRZ } \\
(\boldsymbol{n}=\mathbf{1 4})\end{array}$ & $\begin{array}{c}\text { MEX } \\
(\boldsymbol{n}=\mathbf{4})\end{array}$ & $\begin{array}{c}\text { TUR } \\
(\boldsymbol{n}=\mathbf{2})\end{array}$ & $\begin{array}{c}\text { RUS } \\
(\boldsymbol{n}=\mathbf{2 0})\end{array}$ \\
\hline $\begin{array}{l}\text { Not available in the hospital/clinic where I practice } \\
\text { High out-of-pocket treatment cost for patient }\end{array}$ & 44 & 21 & 0 & 50 & 0 & 65 \\
$\begin{array}{l}\text { Patient has medical issues why they cannot take the } \\
\text { drug (e.g., cardiac) }\end{array}$ & 42 & 29 & 100 & 25 & 0 & 55 \\
\hline
\end{tabular}


Table 2. Cont.

\begin{tabular}{lcccccc}
\hline In an Adjuvant Setting & $\begin{array}{c}\text { Overall } \\
(\boldsymbol{n}=\mathbf{4 1})\end{array}$ & $\begin{array}{c}\text { U.S. } \\
(\boldsymbol{n}=\mathbf{1 4})\end{array}$ & $\begin{array}{c}\text { BRZ } \\
(\boldsymbol{n}=\mathbf{1})\end{array}$ & $\begin{array}{c}\text { MEX } \\
(\boldsymbol{n}=\mathbf{4})\end{array}$ & $\begin{array}{c}\text { TUR } \\
(\boldsymbol{n}=\mathbf{2})\end{array}$ & $\begin{array}{c}\text { RUS } \\
(\boldsymbol{n}=\mathbf{2 0})\end{array}$ \\
\hline $\begin{array}{l}\text { Use not supported by clinical trial data in this setting } \\
\begin{array}{l}\text { Not included in the formulary of drugs covered by } \\
\text { patients' insurance }\end{array}\end{array}$ & 39 & 64 & 0 & 50 & 100 & 15 \\
$\begin{array}{l}\text { Use not recommended by treatment guidelines or } \\
\text { protocol I follow in this setting }\end{array}$ & 32 & 50 & 0 & 25 & 0 & 25 \\
\hline $\begin{array}{l}\text { In a Metastatic Setting } \\
\text { Overall }\end{array}$ & $\begin{array}{c}\text { U.S. } \\
(\boldsymbol{n}=\mathbf{3 9})\end{array}$ & $\begin{array}{c}\text { BRZ } \\
(\boldsymbol{n}=\mathbf{0})\end{array}$ & $\begin{array}{c}\text { MEX } \\
(\boldsymbol{n}=\mathbf{5})\end{array}$ & $\begin{array}{c}\text { TUR } \\
(\boldsymbol{n}=\mathbf{2})\end{array}$ & $\begin{array}{c}\text { RUS } \\
(\boldsymbol{n}=\mathbf{2 0})\end{array}$ \\
\hline $\begin{array}{l}\text { Not included in the formulary of drugs covered by } \\
\text { patients' insurance }\end{array}$ & 49 & 42 & 0 & 20 & 0 & 40 \\
$\begin{array}{l}\text { Not available in the hospital/clinic where I practice } \\
\text { Use not recommended by treatment guidelines or } \\
\text { protocol I follow in this setting }\end{array}$ & 44 & 42 & 0 & 20 & 0 & 65 \\
\hline
\end{tabular}

a Percentages based only on those physicians who reported that they not so often, rarely or never use trastuzumab. See " $n$ " in each column for the number of physicians used to calculate percentages. ${ }^{\mathrm{b}}$ Barriers reported by $>10 \%$ of these physicians across all markets. U.S., United States; BRZ, Brazil; MEX, Mexico; TUR, Turkey; RUS, Russia.

Nearly one-third of all physicians, and a higher percentage in MEX, BRZ and RUS, answered yes to the question "are there any instances where you have not been able to treat a patient with trastuzumab or have had to delay their treatment due to reimbursement issues" (overall $=31 \%$, U.S. $=10 \%, \mathrm{BRZ}=$ $48 \%, \operatorname{MEX}=37 \%$, TUR $=16 \%$, RUS $=76 \%$ ). The most common reasons for this inability to use trastuzumab or delay in treatment are presented in Table 3. Reimbursement issues were encountered across all clinical settings (overall: neoadjuvant $=28 \%$, adjuvant $=39 \%$, metastatic $=32 \%$ ). The most common setting where reimbursement issues were encountered was the metastatic setting in the U.S. $(60 \%)$ and the adjuvant setting in all other countries $(B R Z=39 \%$, Mexico $=43 \%$, TUR $=33 \%$, RUS $=47 \%)$.

Table 3. Most common reasons physicians had to cancel or delay treatment with trastuzumab (\% of physicians) ${ }^{\mathrm{a}}$.

\begin{tabular}{lcccccc}
\hline \multicolumn{1}{c}{ Reasons for Cancel or Delay } & $\begin{array}{c}\text { Overall } \\
(\boldsymbol{n}=\mathbf{1 5 3})\end{array}$ & $\begin{array}{c}\text { U.S. } \\
(\boldsymbol{n}=\mathbf{2 0})\end{array}$ & $\begin{array}{c}\text { BRZ } \\
(\boldsymbol{n}=\mathbf{3 6})\end{array}$ & $\begin{array}{c}\text { MEX } \\
(\boldsymbol{n}=\mathbf{2 8})\end{array}$ & $\begin{array}{c}\text { TUR } \\
(\boldsymbol{n}=\mathbf{1 2})\end{array}$ & $\begin{array}{c}\text { RUS } \\
(\boldsymbol{n}=\mathbf{5 7})\end{array}$ \\
\hline Patient had no insurance/not eligible for reimbursement & 28 & 32 & 22 & 32 & 39 & 25 \\
Insurance/government refused to fund the treatment & 27 & 32 & 64 & 11 & 24 & 10 \\
Hospital did not have funds to provide trastuzumab & 26 & 10 & 8 & 33 & 8 & 44 \\
Patient unable to pay copayment & 15 & 14 & 6 & 22 & 15 & 16 \\
$\quad$ Other & 4 & 12 & 0 & 2 & 14 & 5 \\
\hline
\end{tabular}

\footnotetext{
${ }^{a}$ Based only on those physicians who reported having to cancel or delay treatment with trastuzumab. U.S., United States; BRZ, Brazil; MEX, Mexico; TUR, Turkey; RUS, Russia.
} 


\subsection{Impact of a Biosimilar to Trastuzumab}

Across all markets, $45 \%$ of physicians reported that they would increase the use of HER 2 antibody therapy if a lower cost biosimilar version of trastuzumab were available (U.S. $=29 \%, B R Z=53 \%$, MEX $=63 \%$, TUR $=23 \%$, RUS $=81 \%$ ). This trend was observed across all clinical settings (Table 4). Among physicians who said a biosimilar version would not increase their use of HER2 antibody therapy, the most common reason was reported as "already always use it in all the appropriate patients/situations" (overall $=35 \%)$.

Table 4. Percentage of physicians who would increase their use of HER2 antibody therapy in different clinical settings if a biosimilar to trastuzumab were available ${ }^{\text {a }}$.

\begin{tabular}{ccccccc}
\hline & $\begin{array}{c}\text { Overall } \\
(\boldsymbol{n}=\mathbf{2 2 3})\end{array}$ & $\begin{array}{c}\text { U.S. } \\
(\boldsymbol{n}=\mathbf{5 8})\end{array}$ & $\begin{array}{c}\text { BRZ } \\
(\boldsymbol{n}=\mathbf{4 0})\end{array}$ & $\begin{array}{c}\text { MEX } \\
(\boldsymbol{n}=\mathbf{4 7})\end{array}$ & $\begin{array}{c}\text { TUR } \\
(\boldsymbol{n}=\mathbf{1 7})\end{array}$ & $\begin{array}{c}\text { RUS } \\
(\boldsymbol{n}=\mathbf{6 1})\end{array}$ \\
\hline $\begin{array}{c}\text { Neoadjuvant setting } \\
\text { Adjuvant setting }\end{array}$ & 46 & 52 & 33 & 53 & 71 & 38 \\
$\begin{array}{c}\text { Duration >6 months } \\
\text { Metastatic setting }\end{array}$ & 45 & 45 & 53 & 43 & 59 & 38 \\
1st line & 50 & 53 & 43 & 53 & 53 & 48 \\
2nd line & 50 & 59 & 38 & 32 & 71 & 57 \\
\hline
\end{tabular}

${ }^{\text {a }}$ Based only on those physicians who reported potential increased use of HER2 antibody therapy if a biosimilar were available. U.S., United States; BRZ, Brazil; MEX, Mexico; TUR, Turkey; RUS, Russia.

\section{Discussion}

This study demonstrates that there are barriers to the use of trastuzumab across the globe due to several physician-identified factors, including access to and cost of treatment. However, almost half of physicians surveyed indicated that the use of HER2-directed therapies would increase with the availability of a lower cost biosimilar HER2 monoclonal antibody.

The healthcare systems for the countries included in this study are varied, though they each offer both government-subsidized and private coverage options for their citizens. The Turkish system is predominantly government-based and managed by the Ministry of Health, though reforms over the past decade have resulted in modestly increased access to private healthcare coverage [17]. Centralized coverage is funded by a social security system that is based on employee, employer and government contributions [17]. In addition, low income citizens receive free basic coverage under the Green Card Scheme [17]. RUS also has a predominantly state-run system, managed by the Ministry of Health and Social Development, which mandates nominally free basic healthcare for all citizens [18]. Funding for this system results from a relatively even mix of tax revenues, payroll contributions and out-of-pocket payments for uncovered services (such as outpatient prescription medicines) [18]. BRZ mandates universal free healthcare for its citizens that is funded at the federal, state and local levels [19]. However, as a result, quality of care varies greatly in different regions of BRZ, and approximately $25 \%$ of Brazilians opt to purchase private medical insurance [19]. The Mexican healthcare system is also a mix of government-subsidized (amount of subsidy based on patient income level) and private coverage [20]. The U.S. healthcare system is based largely on private insurance coverage, purchased 
by the patient, though government-subsidized options are available to older citizens, citizens with certain disabilities and citizens with low income. Recent healthcare reform in the U.S. requires most citizens to purchase some form of basic healthcare coverage [21].

Despite different healthcare systems, barriers to the use of trastuzumab were identified in every country examined. Though the study was not powered to directly compare different countries, certain assumptions can be made based on the data presented. BRZ, for example, had the least number of patients with government-funded healthcare and the greatest number of patients having to pay for private medical insurance on their own. Likely because of this, physicians from BRZ most often cited "high out-of-pocket costs for the patient" as a barrier to the use of trastuzumab. Though $\sim 70 \%$ of patients in MEX and RUS had government-funded healthcare, physicians in these countries most often reported that trastuzumab was "not available in the hospital/clinic where they practice" or "not included in the formulary of drugs covered by patient's insurance" as barriers to the use of trastuzumab. Not surprisingly, physicians from BRZ, MEX and RUS most often reported having to cancel or delay treatment with trastuzumab due to reimbursement/payment issues. Even in the U.S., where private medical insurance is predominate, physicians commonly reported "high out-of-pocket costs for the patient", trastuzumab "not being included in the formulary of drugs covered by patient's insurance" or trastuzumab "not being available in the hospital/clinic where they practice" as barriers to the use of trastuzumab. Overall, these data demonstrate that the cost of treatment is a significant barrier to the access and use of trastuzumab. In addition, clinical treatment guidelines were often cited as a barrier to the use of trastuzumab, particularly in an adjuvant or metastatic treatment setting.

The costs of cancer care are increasingly becoming important to the U.S. payer system, as cost containment is a key component of the Affordable Care Act [15]. A study comparing the treatment cost of biosimilars $v s$. innovator biologic for five common treatments in India estimated that the use of biosimilars would result in yearly savings of $\sim 843$ million U.S. dollars [22]. Reditux ${ }^{\mathrm{TM}}$ (Dr. Reddy's Laboratories, Hyderabad, India), for example, the first intended biosimilar to the monoclonal anti-CD20 cancer agent rituximab, was launched in India in 2007 at 50\% lower cost than the originator biologic (MabThera $^{\mathrm{TM}^{\circledR}}$, Hoffman-LaRoche, Basel, Switzerland). As a result, patient access to CD-20-directed therapy increased six-fold within three years of launch [23]. Likewise, the availability of a trastuzumab biosimilar may alleviate the cost-related barriers to the access and use of HER2 antibody therapy. Many oncologists clearly understand the cost benefit of biosimilars, as a majority of those surveyed from the U.S. and the EU expect to prescribe biosimilars to common monoclonal antibody treatments within the first 12 months of their launch [24].

In addition to HER2+ breast cancer patients, other patient populations may also benefit from the availability of a biosimilar to trastuzumab. Trastuzumab is also indicated for the treatment of HER2-overexpressing metastatic gastric or gastroesophageal junction adenocarcinoma, and its addition to chemotherapy significantly improves overall survival [25]. In the future, these patients may also benefit from the availability of a biosimilar to trastuzumab. However, the current study does not address the use of trastuzumab or the availability of a biosimilar in these malignancies.

All survey research, including the current study, is limited in some way. For example, respondents (physicians in this case) may not precisely recall experiences in the past. Additionally, the survey question regarding the use of a potential biosimilar to trastuzumab is limited in that it does not specify exactly how much less expensive the biosimilar would be compared to Herceptin ${ }^{\circledR}$. In order to achieve 
our survey responder goal of 500 physicians (200 U.S. and 75 each in MEX, BRX, RUS and TUR), nearly 8,000 total physicians were invited to participate. It is possible that our findings are subject to non-responder bias, since a majority of invited physicians did not participate or were unable to participate due to closure of the survey once the responder goal was reached. Though the physicians and countries included in this survey vary in their geographic, economic, cultural and healthcare environment, care must be taken when attempting to generalize our findings to physicians from countries outside the scope of this study.

\section{Conclusions}

Innovations in cancer therapy are costly and contribute to rising healthcare expenditures. Improved accesses to therapies and novel delivery models have the potential to advance the prevention and treatment of cancer. The introduction of high-quality biosimilars could potentially benefit patients by broadening access to alternative biologic therapies. In this study, we conclude that there are significant barriers to the use of trastuzumab and that a biosimilar to trastuzumab could increase access to and use of HER2-directed antibody therapy in patients with HER2+ breast cancer.

\section{Acknowledgments}

This study was sponsored by Pfizer Inc. Editorial/medical writing support was provided by Matt Soulsby, PhD, of Engage Scientific Solutions and was funded by Pfizer Inc. The authors thank Sarah Scott, PhD, of Pfizer Inc for her contributions to the development of the physician questionnaire.

\section{Author Contributions}

P.L., C.C., and G.C. contributed to the interpretation of data and drafting of the manuscript. I.J. contributed to the design of the study, interpretation of data, and drafting of the manuscript.

\section{Conflicts of Interest}

P.L. has worked as a consultant for Boehringer Ingelheim over the last three years and will participate in an advisory board for Pfizer this year. C.C. and G.C. have no conflicts of interest to declare. I.J. is a full-time employee of Pfizer Inc.

\section{References}

1. International Agency for Research on Cancer (IARC) and World Health Organization (WHO). GLOBOCAN 2012: Estimated Cancer Incidence, Mortality and Prevalence Worldwide in 2012. Available online: http://globocan.iarc.fr/Pages/fact_sheets_cancer.aspx (accessed on 18 March 2014).

2. Siegel, R.; Ma, J.; Zou, Z.; Jemal, A. Cancer statistics, 2014. CA Cancer J. Clin. 2014, 64, 9-29.

3. Coleman, M.P.; Quaresma, M.; Berrino, F.; Lutz, J.M.; de Angelis, R.; Capocaccia, R.; Baili, P.; Rachet, B.; Gatta, G.; Hakulinen, T.; et al. Cancer survival in five continents: A worldwide population-based study (CONCORD). Lancet Oncol. 2008, 9, 730-756.

4. World Health Organization (WHO). Breast Cancer: Prevention and Control. Available online: http://www.who.int/cancer/detection/breastcancer/en/index1.html (accessed on 6 February 2014). 
5. Genentech. Herceptin US prescribing information. Available online: http://www.gene.com/ download/pdf/herceptin_prescribing.pdf (accessed on 6 February 2014).

6. European Medicines Agency. Herceptin EU Prescribing Information. Available online: http://www.ema.europa.eu/docs/en_GB/document_library/EPAR_-

_Product_Information/human/000278/WC500074922.pdf (accessed on 18 March 2014).

7. Piccart-Gebhart, M.J.; Procter, M.; Leyland-Jones, B.; Goldhirsch, A.; Untch, M.; Smith, I.; Gianni, L.; Baselga, J.; Bell, R.; Jackisch, C.; et al. Trastuzumab after adjuvant chemotherapy in HER2-positive breast cancer. N. Engl. J. Med. 2005, 353, 1659-1672.

8. Romond, E.H.; Perez, E.A.; Bryant, J.; Suman, V.J.; Geyer, C.E., Jr.; Davidson, N.E.; Tan-Chiu, E.; Martino, S.; Paik, S.; Kaufman, P.A.; et al. Trastuzumab plus adjuvant chemotherapy for operable HER2-positive breast cancer. N. Engl. J. Med. 2005, 353, 1673-1684.

9. Slamon, D.J.; Leyland-Jones, B.; Shak, S.; Fuchs, H.; Paton, V.; Bajamonde, A.; Fleming, T.; Eiermann, W.; Wolter, J.; Pegram, M.; et al. Use of chemotherapy plus a monoclonal antibody against HER2 for metastatic breast cancer that overexpresses HER2. N. Engl. J. Med. 2001, 344, 783-792.

10. Gianni, L.; Pienkowski, T.; Im, Y.H.; Roman, L.; Tseng, L.M.; Liu, M.C.; Lluch, A.; Staroslawska, E.B.; de la Haba-Rodriguez, J.; Im, S.A.; et al. Efficacy and safety of neoadjuvant pertuzumab and trastuzumab in women with locally advanced, inflammatory, or early HER2-positive breast cancer (NeoSphere): A randomised multicentre, open-label, phase 2 trial. Lancet Oncol. 2012, 13, 25-32.

11. Schellekens, H. Biosimilar therapeutics-what do we need to consider? NDT Plus 2009, 2, i27-i36.

12. Zelenetz, A.D.; Ahmed, I.; Braud, E.L.; Cross, J.D.; Davenport-Ennis, N.; Dickinson, B.D.; Goldberg, S.E.; Gottlieb, S.; Johnson, P.E.; Lyman, G.H.; et al. NCCN biosimilars white paper: Regulatory, scientific, and patient safety perspectives. J. Nat. Compr. Canc. Netw. 2011, 9, S1-S22.

13. Cai, X.; Wake, A.; Gouty, D. Analytical and bioanalytical assay challenges to support comparability studies for biosimilar drug development. Bioanalysis 2013, 5, 517-520.

14. Megerlin, F.; Lopert, R.; Taymor, K.; Trouvin, J.H. Biosimilars and the European experience: Implications for the United States. Health Aff. (Millwood) 2013, 32, 1803-1810.

15. Patient Protection and Affordable Care Act of 2009, Pub. L. No. 111-148, Title VII, Improving access: Biologics Price Competition and Innovation Act of 2009, Section 7001-7003.

16. European Medicines Agency. Concept Paper on the Revision of the Guideline on Similar Biological Medicinal Product. Available online: http://www.ema.europa.eu/docs/en_GB/document_library/ Scientific_guideline/2011/11/WC500117987.pdf (accessed on 18 March 2014).

17. Tatar, M.; Mollahaliloglu, S.; Sahin, B.; Sabahattin, A.; Maresso, A.; Hernandez-Quevedo, C. Turkey Helath System Review. Health Syst. Transit. 2011, 13, 1-186.

18. Popovich, L.; Potapchik, E.; Shishkin, S.; Richardson, E.; Vacroux, A.; Mathivet, B. Russian Federation Health System Review. Health Syst. Transit. 2011, 13, 1-190.

19. Paim, J.; Travassos, C.; Almeida, C.; Bahia, L.; Macinko, J. The Brazilian health system: History, advances, and challenges. Lancet 2011, 377, 1778-1797.

20. The Organisation for Economic Co-operation and Development. OECD Reviews of Health Systems: Mexico. Available online: http://www.borderhealth.org/files/res_839.pdf (accessed on 21 July 2014). 
21. Patient Protection and Affordable Care Act of 2009, Pub. L. No. 111-148.

22. Lopes, G. Cost comparison and economic implications of commonly used originator and generic chemotherapy drugs in India. Ann. Oncol. 2013, 24, v13-v16.

23. Qureshi, Z.P.; Magwood, J.S.; Singh, S.; Bennett, C.L. Rituximab and biosimilars-Equivalence and reciprocity. Biosimilars 2013, 3, 19-25.

24. Merron, A. Biosimilars advisory Service: Physician Perspectives on G-CSFs and Monoclonal Antibodies in Oncology Available online: http://bio-trends.com/Products-andServices/Report?r=basxxx0113 (accessed on 19 March 2014).

25. Bang, Y.J.; van Cutsem, E.; Feyereislova, A.; Chung, H.C.; Shen, L.; Sawaki, A.; Lordick, F.; Ohtsu, A.; Omuro, Y.; Satoh, T.; et al. Trastuzumab in combination with chemotherapy vs. chemotherapy alone for treatment of HER2-positive advanced gastric or gastro-oesophageal junction cancer (ToGA): A phase 3, open-label, randomised controlled trial. Lancet 2010, 376, $687-697$.

(C) 2014 by the authors; licensee MDPI, Basel, Switzerland. This article is an open access article distributed under the terms and conditions of the Creative Commons Attribution license (http://creativecommons.org/licenses/by/3.0/). 